\title{
Abdominal injuries and sport
}

\author{
John M Ryan
}

\section{Introduction}

Serious abdominal trauma as a result of sporting activities is an uncommon problem. However, because of its relative infrequency, injuries may sometimes go undiagnosed. Significant blunt abdominal injuries do not present in the same way as major orthopaedic injuries, usually because these injuries are, for the most part, internal and concealed. Thankfully penetrating injuries are quite rare and usually confined to high velocity sports when they are sustained by impaling on a stationery object. Penetrating injury from sports such as fencing and shooting are rare, as the potential risk is recognised and appropriate preventive measures are taken by the responsible sporting bodies.

Like so many other sporting injuries with serious consequences, significant trauma is often considered only when the medical attendant has a high index of suspicion. It is important therefore that the forces involved in an injury and the mechanism of the injury are understood, and to have some knowledge of the sport or activity during which the injury was sustained.

This paper will review the causes of abdominal injuries in sport. It will describe patterns of abdominal injuries associated with different sports and describe symptoms and signs associated with these injuries, and their initial management, investigation, and definitive management. In addition, it will review areas in which injury prevention can play a significant role.

Sports associated with abdominal trauma Numerous sports have been implicated in the medical literature in causing abdominal trauma. While fencing, shooting, and cycling constitute the sports most likely to be associated with penetrating injuries, serious blunt injuries are more commonly found in contact and high velocity sports such as football and equestrian and aerial sports.
Main sports implicated in abdominal trauma
- Skiing
- Cycle and motorcycle events
- Equestrian events
- Football
- Mountain climbing

Serious abdominal injuries caused by sporting activities are on the increase. A review of children admitted to hospital with abdominal injuries over a 30 year period has shown that sport is an increasingly common cause, the most common being a bicycle accident. ${ }^{1}$

Motorcycle sports are particularly associated with blunt trauma sustained in high speed accidents. Motorcycle sports are becoming increasingly popular among young people, and, although the severity of injuries sustained varies, cases of ruptured liver have been described. ${ }^{2}$ Mountain bike riding has been shown to cause both liver tears and subcapsular haematomas from handlebar ends, transection of the pancreas, and small bowel evisceration. $^{34}$

Ruptured stomach has been reported to result from diving accidents. Although a rare event, it is important to rule out air embolism and decompression sickness before surgery. ${ }^{56}$ It may be a complication of brief or deep dives, and the possibility must be considered for divers with abdominal symptoms and signs.

\section{Unusual injuries}

A number of unusual but serious abdominal injuries have been sustained during sporting activities including serious abdominal injury from a stingray attack, ${ }^{7}$ an acute abdomen in a swimmer caused by an abdominal wall haematoma, ${ }^{8}$ a bochdalek hernia with incarceration of the transverse colon in the left thoracic cavity in an adult after a tug of war competition, ${ }^{9}$ traumatic rupture of the abdominal wall in an ice hockey player caused by a blunt stab from the end of a stick, ${ }^{10}$ anaphylactic shock in a footballer with a ruptured hydatid liver after blunt trauma to the abdomen from a football, ${ }^{11}$ and aortic rupture after blunt abdominal trauma while playing football. ${ }^{12}$

Occult gastrointestinal bleeding has been shown to occur in long distance runners, a strong correlation with lower gastrointestinal symptoms also being observed. ${ }^{13}$ Severe internal injuries have been reported to result from a fall against the uncapped suction drain of a paddling pool. ${ }^{14}$ Spontaneous rupture of the left hepatic duct has been described in a 24 year old man after an intensive muscle building exercise. $^{15}$ 
Anatomy

The upper abdomen contains the liver and spleen, the organs most at risk of causing haemorrhagic shock as a result of blunt abdominal trauma. Indeed the risk for athletes may be greater than normal. It has been shown by ultrasound that the diameter of the left and right lobes of the liver and the diameter of the arteries in the abdomen are larger in well trained endurance athletes than a matched group of moderately trained endurance athletes and a group of sedentary male subjects. ${ }^{16}$

Blunt abdominal trauma causing jejunal rupture has been described following a motorcycle accident and a punch to the abdomen as well as following blunt trauma to the abdomen in footballers. ${ }^{17-19}$ The subdued clinical presentation and non-specific laboratory findings make diagnosis difficult. The injury is associated with high morbidity and mortality if diagnosis is delayed, and it is a prime example of why the doctor should err on the side of caution with injury to the abdomen. Persistent pain is a particularly common symptom.

The left hemidiaphragm is particularly vulnerable to tears resulting in herniation, an injury that is notorious for late presentation. Indeed fractures of the lower ribs should also be considered, not only as an indicator of hepatic or splenic damage but also of pulmonary contusion and possible pleural damage.

The retroperitoneal space contains organs that are particularly vulnerable to sudden deceleration injuries such as occur in motorcycle accidents. Transection of the aorta is an injury associated with sudden deceleration and is deemed unsurvivable; however, partial tears may respond to conservative treatment or surgery. Suspicion of the injury based on the mechanism of injury is the most critical factor. Injuries that are less life threatening include damage to the pancreas, duodenum, and inferior vena cava. Retroperitoneal haemorrhage following blunt trauma is a particularly difficult injury to identify and may lead to significant hypovolaemia. Damage to the kidneys, ureters, and colon are usually less severe in sports injuries but nevertheless a misdiagnosis can have serious consequences.

The pelvic cavity and its contents are usually well protected from sports injuries except those sustained in high velocity sports such as motor sports and mountain climbing; high impact injuries can be life threatening. Fractures of the pelvis can be fatal if associated haemorrhage goes unrecognised. The bladder may be damaged by comminuted fractures of the pelvis as may the rectum, iliac vessels, and internal genitalia in the female.

Indeed the abdominal wall itself is not an uncommon site for injury, particularly in tennis players and volleyball players; the mechanics of the ground stroke and overhead volley predisposes participants in these sports to chronic abdominal wall injuries. ${ }^{20}$

\section{Mechanism of injury}

Understanding the mechanism of an injury is paramount in diagnosing the injury sustained. This is particularly relevant in cases of blunt
Clinical tips

- Understand the mechanism of injury

- Maintain a high index of suspicion for unusual injuries

- Adhere to ABC principles in resuscitation of the shocked patient

- Consider pregnancy in the injured female athlete

- Significant injuries may have a delayed presentation

abdominal trauma. It is also important that information about possible injuries that may have been sustained is conveyed to those providing definitive care. Witnesses of accidents should convey information to staff at emergency departments particularly if those staff may be ignorant of a particular sport or the nature of forces involved in that sport. Increasingly ambulances carry polaroid cameras to take photographs of injury scenes. These can act as a valuable resource in communicating information about the mechanism of an injury.

One must always suspect solid organ rupture including the liver and spleen in blunt abdominal trauma. Similarly one must consider the possibility of the pregnant uterus now that athletes are participating in competitive contact sports to the second and third trimesters of pregnancy. Shearing injuries to solid organs are found when restraining devices such as seat belts have been used. Sudden deceleration injuries may result in liver and spleen lacerations at the sites of supporting ligaments. Penetrating wounds traverse adjacent abdominal organs and most commonly involve the liver, small bowel, diaphragm, and colon. Although rupture of a major vessel can lead to rapid loss of blood, insidious blood loss can result from apparently insignificant injury of the spleen, liver, or kidney and result in a delayed presentation. Awareness of the potential for such injury is important among players, coaches, and medical attendants at sports events, as outcome can be adversely affected by a low index of suspicion. Ignorance of sporting injuries may compound an injury, as the initial physical examination may not always be a reliable indcator of the severity. ${ }^{21}$

Hollow visceral injuries can also cause problems - for example, mechanical trauma in long distance runners, the so-called "caecal slap syndrome", can result in repeated microtrauma of the caecum against a hypertrophied muscle wall causing colic. ${ }^{22}$

\section{The approach to management}

Increasingly doctors manage life threatening injury according to the teaching of the American College of Surgeon's Advanced Trauma Life Support course. If there are multiple injuries, the assessment and management of abdominal injuries must take second place to potentially more life threatening ones. Thus, airway and cervical spine control and ventilation must take priority over circulation and control of haemorrhage, which are usually the 
most serious consequences of severe abdominal injury. However, multiple injuries in sport are unusual and the field medical officer, primary care practitioner, or emergency department doctor is more likely to be confronted with blunt abdominal trauma in the presence of normal physiological variables than with an unstable patient.

The assessment and management of the patient with abdominal trauma will vary depending on the environment. The priorities for the immediate care doctor are different from those of the emergency room physician who, unlike the former, has access to a greater array of diagnostic and therapeutic procedures.

\section{Initial assessment}

It is important to understand the mechanism of how an injury has been sustained and the anatomy of the abdomen. It is equally important to identify the signs associated with intraperitoneal, retroperitoneal, and pelvic trauma, to apply diagnostic and therapeutic procedures specific to abdominal trauma, and to understand the limitations and advantages of available diagnostic procedures.

The immediate assessment of the athlete with abdominal injury must take into account the potential for the evolution of critical injuries. The traditional medical model for examination of the abdomen, including inspection, palpation, percussion, and auscultation, may be valuable. In patients who are hypotensive, transfer to early definitive care must be a priority while recognising that management of the airway and ventilation must take priority if indicated. Care must also be taken to protect the cervical spine in the presence of multiple injuries. Assessment of the back should be carried out during a meticulous log roll. Involuntary guarding and rebound tenderness should be sought as signs of peritoneal irritation. Percussion may elicit signs of peritonitis, gastric dilatation, or haemoperitoneum. The absence of bowel sounds may reveal an ileus caused by injuries to adjacent structures.

Examination of the external genitalia should not be forgotten, looking for blood at the external urethral meatus and inspection of the scrotum and perineum. Rectal examination may be indicated to look for evidence of a high riding prostate which may be associated with a fractured pelvis and also to evaluate the presence of bony fragments.

\section{Resuscitation}

The resuscitative phase, once the airway and ventilation have been dealt with, includes venous access and commencement of intravenous crystalloid fluids. Blood should be sent for group and cross match. A nasogastric tube relieves gastric dilatation and reduces the risk of gastric aspiration. Insertion of a urinary catheter, provided there is no evidence of urethral trauma, can act as an additional monitoring tool in resuscitation. Repeated examinations are particularly important for injuries such as gastrointestinal tract perforations, which may be difficult to diagnose.
Early review by senior qualified staff remains an important critical step in evaluation of the athlete with serious abdominal injury. It may be important to alert an emergency department in advance of a patient's arrival so that a trauma team can be mobilised to evaluate a patient on arrival and minimise the time to critical intervention if surgery is required. Death from haemorrhagic shock is a tragedy and often preventable. It should never result from a sporting injury. Medical officers responsible for athletes involved in high impact or contact sports should understand how a modern emergency department functions and have direct access to nominated senior staff at the local emergency department from an event or competition site.

\section{Investigation}

Patients with significant blunt abdominal trauma should have baseline haematological and biochemical tests performed including a full blood count and determination of electrolytes, glucose, amylase, and, in the female athlete, human chorionogonadotrophic hormone to determine pregnancy. It may be best to determine the amylase level three hours after the injury to avoid failure in detection of pancreatic injury. ${ }^{23}$ Plain radiography is not very useful but may disclose pneumoperitoneum or obliteration of the psoas shadow, indicating a retroperitoneal haematoma.

Diagnostic imaging is the most important tool in the assessment of the athlete with abdominal injury. Contrast radiography with or without computed tomography and ultrasonography are the most widely used tools. Diagnostic peritoneal lavage may be rapidly performed but it is an invasive procedure. With the increasing availability of bedside ultrasonography and rapid access to computed tomography, it is likely that we will see a decrease in the frequency with which it is used. Indeed ultrasonography has been shown to have equal sensitivity and specificity to diagnostic peritoneal lavage in diagnosing intraperitoneal free fluid. Ultrasonography has the added advantage of being a virtually dynamic investigation and can be repeated at frequent intervals. Although particularly useful for liver and splenic injuries, its sensitivity for identifying pancreatic and intestinal injuries is considerably lower. ${ }^{24}$ In children who have sustained blunt abdominal trauma, ultrasonography alone may be sufficient for assessing the abdomen, although patients with more than minimal free intraperitoneal fluid should also have a computed tomographic examination. ${ }^{25}$ Use of computed tomography has been shown to increase the non-operative management of splenic trauma. ${ }^{26}$ More advanced imaging may be required for pancreatic injuries. Double contrast computed tomography has been suggested for patients with abdominal pain, increased amylase activity, and a handlebar injury. ${ }^{27}$ Serial physical examinations have been shown to be equally sensitive in detecting early intra-abdominal haemorrhage and should be performed regularly in the patient with suspected intra-abdominal pathology. 
Genitourinary injuries are by far the most common injuries to the abdomen sustained in sporting activity. They usually result from a direct blow to the back or flanks resulting in contusions, haematomas, or localised bruising. Injury is often associated with frank or microscopic haematuria. The presence of frank haematuria after abdominal injury and microscopic haematuria with shock or microscopic haematuria and hypotension mandates imaging of the renal tract. ${ }^{28}{ }^{29}$ Whether it is contrast imaging or ultrasonography is largely irrelevant and often depends on local skills and availability of resources.

Winter sports, particularly downhill skiing, are gaining in popularity. Improved technology has allowed faster speeds to be attained and access to more difficult terrain, both of which result in increased numbers of abdominal and urology related injuries. However, there is no characteristic pattern of ski related urological injuries other than those associated with major blunt abdominal trauma. Injuries of the lower urinary tract are relatively uncommon. ${ }^{30}$ One centre has described a high incidence of nephrectomy $(21 \%)$ among patients admitted with renal trauma resulting from skiing accidents over a 19 year period. ${ }^{31}$ Sledding injuries have also been reported on occasions to cause abdominal injuries requiring hospitalisation. ${ }^{32}$ Another report described more severe splenic injuries in association with renal trauma occurring as a result of high speed collision when compared with injuries sustained by falling on moguls or rocks. This latter group are more likely to ski down a mountain side themselves and present late, often after frank haematuria has been noticed by the skier. It is important to remember that those who ski down the mountain themselves and present late may still have serious abdominal injury and with a potentially higher rate of splenic salvage. ${ }^{33}$

Haematuria from bladder injury can occur when the posterior bladder wall repeatedly impacts against the bladder base causing vascular lesions. ${ }^{34}$ Blood at the external urethral meatus or perineal bruising should always raise the suspicion of pelvic fractures.

\section{Definitive care}

Once the diagnosis of haemoperitoneum is made, a decision must be taken about whether to proceed to surgery. Several studies have shown that non-operative management of liver and spleen injuries may be successful. The ruptured spleen, particularly in children, is more often being managed conservatively as the importance of the spleen becomes increasingly recognised. The risk of overwhelming post-splenectomy infection is a real concern. Nowadays it is rare indeed for children with splenic injury to undergo surgery, particularly when the spleen is the only organ injured and no other major intra-abdominal injuries have occurred.

Indeed even the non-operative management of extensive hepatic and splenic injuries with significant haemoperitoneum may be successful. $^{35}$ Factors that may indicate laparotomy include hypotension in the pre-

\section{Key points}

- Serious injuries are rare

- Understanding mechanisms of injury is important

- Symptoms must be taken seriously

- Advanced diagnostic imaging is valuable

- Resuscitation should follow ABC principles

- Sporting bodies have a role to play in injury prevention

hospital setting or the emergency department, tachycardia, abnormal packed cell volume (less than 30) or coagulopathy, or blood transfusion in the emergency department. ${ }^{36}$

Most renal injuries are treated conservatively. Patients with normal imaging results can be treated with rest and follow up urinalysis to ensure clearing of haematuria. Those with signs of significant extrarenal leakage on urography, angiography, or computed tomography should receive immediate surgical management. ${ }^{37}$

The treatment of blunt pancreatic trauma remains controversial. Morbidity is higher when there is main pancreatic duct involvement, and delay in diagnosing this injury may lead to significant morbidity or death. Parenchymal injuries not involving the ductal system rarely result in significant morbidity or death. Thus surgical intervention should depend on the integrity of the main pancreatic duct. ${ }^{38}$

\section{Injury prevention}

The bodies responsible for the laws of a sport have an obligation to address injury prevention when patterns of injury become apparent, and should take appropriate steps to counteract injury profiles with recurrent patterns. McLatchie first suggested this in 1981 in his paper on karate injuries in which he proposed outlawing certain uncontrollable methods of attack and mandatory provision of protective equipment such as padded floors. ${ }^{39}$ Despite the knowledge of injury patterns, effecting change remains extremely difficult, particularly in sports such as skiing where participants are frequently young 'risk takers'. Even with increasing awareness of injury patterns, Furrer et $a l^{40}$ reported no improvements and in fact reported an increase in the mortality rate for patients with severe skiing injuries between 1989 and 1992 when compared with the period 1984-1988, although those who sustained abdominal or thoracic injuries had the best prognosis. Prall et $a l^{41}$ have also shown that rates of serious injuries from skiing are similar to those for snowboarding, although splenic injuries are more common in snowboarders. The rate for snowboarders requiring tertiary referral is estimated at 0.03 injuries/1000 snowboarder days, a risk that should be conveyed to the public.

The importance of injury surveillance and reporting of emerging sports has been shown to be highly relevant to abdominal injury in sport. In 1976 Sturz et $a l^{42}$ reported an increasing number of injuries from the use of skateboards, 
including fatalities from abdominal injuries, and was able to make recommendations about their use in public streets.

As new sports and new variants of sports develop, so too new injury patterns develop about which the public need to be educated. Sacco $e t a l^{43}$ have described how splenic injuries are now being seen more commonly in snowboarders: in their study, splenic injuries were six times more likely in boarders than skiers, although the younger age of participants in this sport perhaps reflected the low operative rate, only two splenectomies.

In horse riding the importance of checking tack has been shown in one paper in which tack failure was found to cause several injuries. In a prospective study of 110 cases, there were four renal contusions and one bladder laceration. ${ }^{44}$

In children, life threatening abdominal injuries have been shown to be associated with handlebar trauma when the handlebars had no foam or plastic coating covering the metal ends. Some form of padded protection of handlebar ends is recommended unless the design can be suitably modified, as it has been suggested that the damage is caused by handlebars with a small cross-sectional diameter at the end. ${ }^{45}{ }^{46}$ Furthermore, subjects with handlebar injuries often present late, with serious consequences. ${ }^{47}$

Preparedness is equally important in terms of preventing deterioration. The value of air evacuation for patients sustaining high speed abdominal injuries during skiing has been shown by Jurkovich et $a l^{48}$ who reported a series of patients for whom resuscitation was initiated on arrival of a helicopter and who were then taken directly to a trauma centre for definitive care.

Evidence also suggests that prevention of less serious injuries may also be possible. Biomechanical changes have been shown to occur in the erector spinae and abdominal oblique muscles of golfers during a golf swing, suggesting the need for an effective preventive exercise programme. $^{49}$

\section{Consequences}

The consequences of blunt abdominal injury in sport extend beyond physical morbidity. In terms of lost time from work or school, the consequences are high. Even financially these preventable injuries may have a significant impact. Shorter et al in 1996 reported the average cost of skiing injuries to children and adolescents to be $\$ 22000$ in a series that included six patients with abdominal injuries. ${ }^{50}$

\section{Summary}

Serious abdominal injuries resulting from sport are rare. The potential for misdiagnosis is significant and the consequences may be serious. Patients with abdominal pain should be taken very seriously and investigated with appropriate diagnostic equipment. Sporting bodies have a responsibility to address safety within a particular sport and to change the rules where necessary as injury patterns are identified.
1 Bergqvist D, Hedelin $\mathrm{H}$, Lindblad B, et al. Abdominal Injuries in children: an analysis of 348 cases. Injury ries in childre

2 Fontijne WP, Duis HJ, Klasen HJ. Motor cycle injuries in speed sport for youngsters. F Sports Med Phys Fitness 1989; 29:199-201.

3 Lovell ME, Brett M, Enion DS. Mountain bike injury to the abdomen, transection of the pancreas and small bowel evisceration. Injury 1992;23;449-500.

4 Nehoda H, Hochleitner BW. Subcapsular liver haematomas caused by bar ends in mountain-bike crashes. Lancet 1998; 351:342.

5 Molenat FA, Boussuges AH. Rupture of the stomach complicating diving accidents. Undersea Hyperb Med 1995;22: $87-96$.

6 Cramer FS, Heimbach RD. Stomach rupture as a result of gastrointestinal barotrauma in a scuba diver. $\mathcal{f}$ Trauma $1982 ; 22 ; 238-40$

7 Cross TB. An unusual stingray injury: the skindiver at risk. Med F Aust 1976;2:947-8.

8 Stattin J, Persson K, Wai D. Acute abdomen in a swimmer was caused by abdominal wall haematoma. Lakartidningen 1992;89:2689.

9 Lai T, Ohmori K, Mishina T, et al. Adult bochdalek hernia after playing at a tug of war. Kyobu Geka 1997;50:968-70.

10 Zaunschirm G, Steiner E. Traumatic rupture of the abdominal wall: an injury in ice hockey. Sportverletz Sportschaden 1989;3:187-8.

11 Yahya AI, Przybylski J, Foud A. Anaphylactic shock in a patient with ruptured hydatid liver cyst owing to trivia abdominal trauma. F R Coll Surg Edinb 1997;42:423-4

12 Lyons AR. Aortic rupture as a result of a sporting injury. Ann Emerg Med 1992;21:425-7.

13 Baska RS, Moses FM, Graeber G, et al. Gastrointestinal bleeding during an ultramarathon. Dig Dis Sci 1990;35: $276-9$.

14 Centers for Disease Control. Suction-drain injury in a public wading pool, North Carolina, 1991. fAMA 1992;267: 2868 .

15 Muller MF, Krestin GP, Weder W, et al. Spontaneous rupture of the left hepatic duct. Dtsch Med Wochenschr rupture of the left

16 Gabriel H, Kindermann W. Ultrasound of the abdomen in endurance athletes. Eur f Appl Physiol 1996;73:191-3.

17 Harris CR. Blunt abdominal trauma causing jejunal rupture. Ann Emerg Med 1985;14:916-18.

18 Lindemann JM, Schmid D, Akovbiantz A. Jejunum perforation following blunt abdominal trauma: a case report. Schweiz Rundsch Med Prax 1994;83;857-60.

19 Murphy CP, Drez D. Jejunal rupture in a football player. $\mathrm{Am}$ 7 Sports Med 1987;15;184-5.

20 Balduini FC. Abdominal and groin injuries in tennis. Clin Sports Med 1988;7;349-57.

21 Stricker PR, Hardin BH, Puffer JC. An unusual presentation of liver laceration in a 13-yr-od football player. Med Sci
tion tion of liver laceration in a 13
Sports Exerc 1993;25;667-72.

22 Michel H, Larrey D, Blanc P. Hepato-digestive disorders in athletic practice. Presse Med 1994;23;479-84.

23 Takishima T, Sugimoto K, Hirata M, et al. Serum amylase level on admission in the diagnosis of blunt injury to the pancreas: its significance and limitations. Ann Surg 1997;226:70-6.

24 Yoshii H, Sato M, Yamamoto S, et al. Usefulness and limitations of ultrasonography in the initial evaluation of blunt abdominal trauma. 7 Trauma 1998;45:45-50.

25 Akgur FM, Aktug T, Olguner M, et al. Prospective study investigating routine usage of ultrasonography as the initial diagnostic modality for the evaluation of children
intic sustaining blunt abdominal trauma. F Trauma 1997;42: 626-8.

26 Brasel KJ, DeLisle CM, Olson CJ, et al. Splenic injury: trends in evaluation and management. $\mathcal{F}$ Trauma 1998;44: 283-6.

27 Arkovitz MS, Johnson N, Garcia VF. Pancreatic trauma in children: mechanisms of injury. F Trauma 1997;42:49-53.

8 Miller KS, McAninch JW. Radiographic assessment of rena trauma: our 15-year experience. F Urol 1995;154:352-5.

29 McAndre JD, Corriere JN. Radiographic evaluation of renal trauma: evaluation of 1103 consecutive patients. Br F Urol 1994;73:352-4.

30 Hildreth TA, Cass AS, Khan AU. Winter sports-related urologic trauma. F Urol 1979;121:62-3.

31 Skowvron O, Descotes JL, Frassinetti E, et al. Kidney injuries due to skiing. Prog Urol 1995;5:361-9.

32 Hedges JR, Greenberg ML. Sledding injuries. Ann Emerg Med 1980;9:131-3.

33 Sartorelli KH, Pilcher DB, Rogers FB. Patterns of splenic injuries seen in skiers. Injury 1995;26:43-6.

34 Abarbanel J, Benet AE, Lask D, et al. Sports hematuria. $\mathcal{F}$ Urol 1990;143;887-90.

35 Goan YG, Huang MS, Lin JM. Non-operative management for extensive hepatic and splenic injuries with significant hemoperitoneum in adults. F Trauma 1998;45:360-4.

36 Cathey KL, Brady WJ, Butler K, et al. Blunt splenic trauma: characteristics of patients requiring urgent laparotomy. $\mathrm{Am}$ Surg 1998;64:450-4.

37 Kristjansson A, Pedersen J. Management of blunt renal trauma. Br f Urol 1993;72;692-6.

38 Bradley EL, Young PR, Chang MC, et al. Diagnosis and initial management of blunt pancreatic trauma: guidelines from a multiinstitutional review. Ann Surg 1998;227:861-9.

39 McLatchie G. Karate and karate injuries. Br 7 Sports Med $1981 ; 15 ; 84-6$. 
40 Furrer M, Erhart S, Frutiger A, et al. Severe skiing injuries: a retrospective analysis of 361 patients including mechanism of trauma, severity of injury, and mortality. F Trauma 1995;39;737-41

41 Prall JA, Winston KR, Brennan R. Severe snowboarding injuries. Injury 1995;26:539-42.

42 Sturz H, Rosemeyer B. Skateboard injuries. $M M W$ Munch Med Wochenschr 1979;121:485-8.

43 Sacco DE, Sartorelli DH, Vane DW. Evaluation of alpine skiing and snowboarding injury in a northeastern state. $\mathcal{F}$ Trauma 1998;44:654-9.

44 Grossman JA, Kulund DN, Miller CW, et al. Equestrian injuries. Results of a prospective study. $\mathcal{F} A M A 1978 ; 240$ : $1881-2$.
45 Acton $\mathrm{CH}$, Thomas S, Clark R, et al. Bicycle incidents in children: abdominal trauma and handlebars. Med $\mathcal{F}$ Aust 994;160:344-6.

46 Clarnette TD, Beasley SW. Handlebar injuries in children: patterns and prevention. Aust N Z F Surg 1997;67;338-9. 7 Sparnon AL, Ford WD. Bicycle handlebar injuries in children. F Pediatr Surg 1986;21:118-19.

48 Jurkovich GJ, Pearce WH, Cleaveland HC. Thoracic and abdominal injuries in skiers: the role of air evacuation. $\mathcal{F}$ Trauma 1983;23;844-8.

49 Pink M, Perry J, Jobe FW. Electromyographic analysis of the trunk in golfers. Am $\mathcal{F}$ Sports Med 1993;21:385-8.

50 Shorter NA, Jensen PE, Harmon BJ, et al. Skiing injuries in children and adolescents. F Trauma 1996;40:997-1001.

\section{Take home message}

Serious abdominal injuries are relatively uncommon in sporting activities. However, the consequences for the athlete when they occur can be life threatening. Athletes, coaches, team doctors, and emergency doctors must be aware of the potential for injuries to occur within a particular sport and understand the significance of symptoms and signs that develop after an injury.

True or false?

1 After blunt trauma to the abdomen sustained during a sports event, athletes suffering from infectious mononucleosis are at increased risk of splenic rupture.

2 Patients with microscopic haematuria should have urgent imaging of the renal tract.

3 An understanding of the mechanism of injury is more likely to result in the correct diagnosis.
4 Rupture of the pancreas is easily diagnosed.

5 In children a ruptured spleen usually requires urgent splenectomy.

(Answers p 222.)

\section{Essay question}

Describe the early management of a footballer complaining of abdominal pain having sustained a kick to the abdomen during a football match. 